\title{
Editorial
}

\section{A Taste of the SfN Annual Meeting}

Each year, JNeurosci publishes a group of review articles based on topics presented in symposia and mini-symposia at the annual meeting of the Society for Neuroscience. These reviews are usually published in a special issue the week before the meeting. Sadly, the 2020 annual meeting was canceled because of the COVID-19 pandemic. This was especially disappointing since the necessity of sharing discoveries and communicating with other scientists is particularly evident during this crisis, and the value of highlighting reliable scientific information has been illustrated again and again. This makes the role of JNeurosci more critical than ever.

Fortunately, many of the symposia and mini-symposia scheduled for 2020 will be presented instead at the 2021 meeting. Rather than wait until next fall to publish the reviews we commissioned, we have decided to publish them now, as a glimpse of some of the topics to be explored at the 2021 annual meeting. As always, these reviews highlight topics of interest across the broad spectrum of neuroscience, from molecules and cells to circuits, brains, behavior, as well as new technological advances. We hope these reviews will whet your appetite for the 2021 SfN annual meeting.

\section{(D) Marina R. Picciotto}

EiC, JNeurosci

https://doi.org/10.1523/JNEUROSCI.0034-21.2021 